\title{
Perspectives
}

\section{Clinical significance of the low normal sperm morphology value as proposed in the fifth edition of the WHO Laboratory Manual for the Examination and Processing of Human Semen}

\author{
Roelof Menkveld
}

Andrology Laboratory, Department of Obstetrics and Gynaecology, Tygerberg Academic Hospital and University of Stellenbosch, Tygerberg 7505, South Africa

\begin{abstract}
The very low cut-off value for sperm morphology of 4\% morphologically normal spermatozoa, as proposed in the new edition of the World Health Organization (WHO) manual on semen analysis, is in agreement with recently published values and reflects the trend of a decline in reported mean values for normal sperm morphology. The reduced value for morphologically normal spermatozoa over the years may be due to several factors. The first is the introduction of strict criteria for the evaluation of sperm morphology. Other reasons may include the introduction of additional criteria for sperm morphology abnormalities and the suggested decrease in semen parameters because of increasing negative environmental influences. Although on its own the newly proposed very low normal value may not provide the strong predictive value for a males' fertility potential, as originally reported for sperm morphology evaluated according to strict criteria, a good predictive value can still be obtained if the holistic, strict approach for sperm morphology evaluation is followed together with additional sperm morphology parameters now available, because certain morphology patterns and sperm abnormalities are now known to be of strong prognostic value. In addition, better international standardization of the technical methodology, consensus on the interpretation of sperm morphology evaluation criteria and standardized international external quality control (EQC) schemes, are of utmost importance to maintain the good predictive value of sperm morphology.
\end{abstract}

Asian Journal of Andrology (2010) 12: 47-58. doi: 10.1038/aja.2009.14

Keywords: male fertility potential, normal cut-off values, semen analysis, sperm morphology evaluation, strict criteria, WHO manual

\section{Introduction}

Reaching a professional consensus always involves a difficult balance of divergent views, and no consensus has been more difficult to reach than that regarding the evaluation and reporting of sperm morphology. Thus, there are sharply differing views on how to assess sperm morphology. This difficulty is reflected in the introduction (Chapter 1) of the 5 th edition of the World Health Organization (WHO)

Correspondence to: Dr Roelof Menkveld, Andrology Laboratory, Department of Obstetrics and Gynaecology, Tygerberg Academic Hospital and University of Stellenbosch, Tygerberg 7505, South Africa.

Fax: +27-21-939-9345

Received: 25 December 2008

Accepted: 23 January 2009
Laboratory Manual for the Examination and Processing of Human Semen [1], which says, on the topic of assessment of sperm morphology, 'Some laboratories assess only normal forms, while others consider the type, location and extent of abnormality to be more important. Whether these or differential or semi-quantitative assessments increase the value of the semen analysis remains contentious. Evidence to support the relationship between the percentage of 'normal' forms, (as defined by strict categorization or computer-aided assessment of morphology) with fertilization rates in vivo, justifies the approach of trying to determine a morphologically distinct sub-population of spermatozoa within semen.'

The introduction goes on to note, 'In the 4th edition, an obvious omission, but inevitable given the lack of data, was the absence of a reference value for the percentage of 
morphologically normal forms; this has been rectified in this manual with the inclusion of data on the percentage of normal forms in a fertile population.' For this purpose, the lower fifth percentile was used, resulting in a very low cut-off value of $4 \%$ morphologically normal spermatozoa. At first impression, this lower reference limit of the percentage of normal spermatozoa in fertile men is so low that it is unlikely that a lower population (from infertile men) could be distinguished from it.

The aim of this article is, therefore, to discuss the usefulness of this very low reference value as well as the biological meaning of such tight categorization of a small minority of spermatozoa, and available tools and data to improve the diagnostic value of the suggested cut-off value.

\section{History of sperm morphology evaluation}

The interest in sperm morphology as a tool in the evaluation of a man's fertility potential started in the early 1900s, when it became accepted that normal and pathological sperm forms could appear simultaneously in a semen sample [2]. Although the importance of normal sperm morphology has been stressed since that time-for example, with statements by Cary in 1916 [3], Williams and Savage in 1925 [4] and Moench and Holt in 1931 [5] that sperm morphology was the single most important source of information on the reproductive potential of spermatozoathe manner in which the normality or abnormality of the spermatozoa should be evaluated has been controversial and remains a field of continuous debate.

The history of sperm morphology evaluation consists of two approaches or phases, which are also reflected in the evolution of sperm morphology evaluation criteria in the consecutive WHO manuals, from the first in 1980 to the soon-to-be-published fifth edition [1, 6-9]. The two approaches are the early, or liberal, approach [10] and the strict (Tygerberg) approach, as introduced by Menkveld in 1987 [11] and described in detail by Menkveld et al. in 1990 [12].

\subsection{Liberal approach}

Human males exhibit extreme heterogeneity, or pleomorphism, of sperm morphology among [13] and even within specific males [14]. This varied morphology contrasts with that of most domestic animals, which generally reveals a very homogeneous sperm population in individual species. This homogeneity for animal sperm morphology made it possible to use the appearance of the modal spermatozoon form, as seen in proven fertile animals, to describe the morphologically normal spermatozoon form for that specific species. Owing to the heterogeneity of human sperm morphology, however, it was not possible to apply this concept successfully in human males. In the typical approach, investigators would describe different obvious abnormalities and use these abnormalities as criteria to evaluate sperm morphology. All spermatozoa not considered abnormal, were regarded as normal, and morphologically normal spermatozoa were, therefore, identified by default. These so-called (remaining) normal spermatozoa as well as specific spermatozoal abnormalities were often depicted by schematic and inaccurate drawings [15]. The liberal approach is illustrated by the words of MacLeod and Gold [16], who stated, 'we are not prepared at this time to classify any but the most distorted forms as truly abnormal.' Owing to the fact that on the basis of this approach no specific criteria were put forward for a morphologically normal spermatozoon, poor correlations could theoretically be expected with normal sperm functions and fertilization rates, as reported by Page and Holding [17].

\subsection{Strict (Tygerberg) criteria}

The strict approach for sperm morphology evaluation was conceptualized in the late 1970 s and early 1980 s, as described by Menkveld in 1987 [11] and Menkveld et al. in 1990 [12]. In contrast to the liberal approach, the definition for a morphologically normal spermatozoon was based on biological evidence obtained from spermatozoa found at the level of the internal cervical os. Spermatozoa found in such samples present with a mostly homogeneous appearing sperm population. Small biological variations in the head morphology can be observed in such samples, but these variations were considered normal. One of the most important aspects of the strict evaluation criteria is that the range allowed for these minute normal biological variations has to be kept as small as possible. For this reason, the socalled 'borderline' or 'slightly abnormal' head forms are regarded as abnormal.

By strict evaluation, the description of a morphologically normal spermatozoon is furthermore supported by the morphological appearance of spermatozoa found tightly bound to the human zona pellucida $[18,19]$.

\subsection{Evolution of WHO criteria for sperm morphology evaluation}

There was no clear textual description for morphologically normal spermatozoa in the 1 st WHO manual published in 1980 [6], except in some legends for the color plates in which it is mentioned that 'the germinal cell classifications are based on those proposed by MacLeod,' thus following the liberal approach. Although slightly improved, the 2nd (1987) WHO manual [7] followed the same liberal approach [13] and provided only a little additional information on sperm morphology evaluation criteria.

In the 3rd (1992) WHO manual [8], the scheme for 
the morphological classification of human spermatozoa was described carefully. The manual stated, 'strict criteria should be applied when assessing the morphological normality of the spermatozoon.' The strict evaluation definition for a morphologically normal spermatozoon is then given, as well as the statement that all 'borderline' forms should be considered abnormal. The 4th (1999) edition of the WHO manual [9] completely adopted the strict (Tygerberg) approach.

\subsection{Evolution of the WHO cut-off point for normal morphology}

Through the progression of the first four WHO manuals, some minor changes occurred in the cut-off values with respect to most semen variables, such as concentration, motility and viability. However, for normal sperm morphology, the values were lowered dramatically from the first edition of the manual published in 1980 to the fourth edition in 1999, and now especially in the fifth edition (Table 1). The first edition [6] included a table with different sperm morphology variables, such as normal, large oval heads and tapering heads, along with mean values and ranges. These values were based on the results of 602 ejaculates from 73 men who were fathers. The mean normal morphology was $80.5 \%$, with a range of $48.0 \%-98.0 \%$. In a footnote to the table, it is stated that, 'Analysis of ejaculate (multiple samples) from at least 50-70 men of recently proven fertility, needs to be carried out at each centre to establish the normal values for a given population, in terms of sperm counts, germinal cell types and their percentages.' Readers were referred to publications by MacLeod [20] and Eliasson [21] for more information. Eliasson [21] considered the morphology cut-off value to be $\geq 60 \%$ morphologically normal spermatozoa.
In the second edition of the WHO manual [7], the normal sperm morphology value was lowered to $50 \%$ morphologically normal forms. In the third edition [8], the value for normal sperm morphology was changed to $\geq 30 \%$ morphologically normal spermatozoa. A note added to this value stated that, 'Although no clinical studies have been completed, experience in a number of centres suggest that the percentage of normal forms should be adjusted downwards when more strict criteria are applied. An empirical reference value is suggested to be $30 \%$ or more with normal forms.' In the fourth edition, issued in 1999 [9], no specific value was indicated for normal sperm morphology, but a note was added stating that 'Multicentre population-based studies utilising the methods of morphology assessment in this manual are now in progress. Data from assisted reproductive technology programmes suggest that, as sperm morphology falls below $15 \%$ normal forms using the methods and definitions described in this manual, the fertilisation rate in vitro decreases.'

In the fifth edition of the WHO manual [1], a new approach has been adopted with regard to the establishment of so-called 'normal,' or reference, values. This new direction is long overdue: many authors have been advocating the change for many years. One of the first groups to advocate these changes was that of the Andrology Unit at Tygerberg Hospital, in the publications by Van Zyl et al. [22, 23], Menkveld and Kruger [24-26], and Menkveld [27].

The proposed value in the fifth edition is very low, $4 \%$ morphologically normal spermatozoa, based on the fifth centile of combined data from several reports in recent publications, in which semen analyses were performed according to known and standardized methodologies [28].

Table 1. Cut-off values for semen variables as published in consecutive WHO manuals [6-9] and as proposed in the fifth World Health Organization (WHO) manual [1].

\begin{tabular}{|c|c|c|c|c|c|}
\hline Semen variable & 1980 & 1987 & 1992 & 1999 & $2010^{1}$ \\
\hline Volume (mL) & - & $\geq 2.0$ & $\geq 2.0$ & $\geq 2.0$ & 1.5 \\
\hline Concentration $\left(10^{6} \mathrm{~mL}^{-1}\right)$ & $20-200$ & $\geq 20$ & $\geq 20$ & $\geq 20$ & 15 \\
\hline Total sperm number $\left(10^{6} /\right.$ ejaculate $)$ & - & $\geq 40$ & $\geq 40$ & $\geq 40$ & 39 \\
\hline Motility (\% motile) & $\geq 60$ & $\geq 50(a+b)^{2}$ & $\geq 50(a+b)$ & $\geq 50(a+b)$ & $40(a+b+c)$ \\
\hline Forward progression (for 1980 only) & $\geq 2^{3}$ & $\geq 25$ (a) & $\geq 25$ (a) & $\geq 25$ (a) & $32(a+b)$ \\
\hline Morphology (\% normal) & $80.5^{4}$ & $\geq 50$ & $\geq 30^{5}$ & $(14)^{6}$ & 4 \\
\hline Viability/vitality (\% live) & - & $\geq 50$ & $\geq 75$ & $\geq 75$ & 58 \\
\hline White blood cells $\left(10^{6} \mathrm{~mL}^{-1}\right)$ & $<4.7$ & $<1.0$ & $<1.0$ & $<1.0$ & $<1.0$ \\
\hline
\end{tabular}

${ }^{1}$ Lower reference limit. Obtained from the lower fifth centile value. ${ }^{2}$ Grade a $=$ rapid progressive motility $\left(>25 \mu \mathrm{m} \mathrm{s}{ }^{-1}\right) ;$ Grade $b=$ slow $/$ sluggish progressive motility (5-25 $\left.\mu \mathrm{m} \mathrm{s}^{-1}\right)$; Grade $\mathrm{c}=$ no-progressive motility; Grade $\mathrm{d}=$ immotility; Normal $=\geq 50 \%$ motility $($ grades a + b) or $\geq 25 \%$ progressive motility (grade a) within $60 \mathrm{~min}$ of ejaculation. ${ }^{3}$ Forward progression on a scale of $0-3$, in which $0=$ no forward progression. ${ }^{4}$ Mean of a fertile population (range $=48 \%-98 \%$ ); modified Bryan Leishman staining. ${ }^{5}$ Arbitrary value. ${ }^{6}$ No actual value given. Multicentre studies in progress refer to $>14 \%$ for in vitro fertilization (IVF). 


\section{Comparison of the proposed WHO value with recently published normal sperm morphology values}

As early as 1975 and 1976, Van Zyl et al. [22, 23] published data proposing much lower normal values on the basis of interval values above which spontaneous pregnancies showed no statistically significant increases with an increase in a particular semen variable value (Table 2). Below these normal cut-off values, two additional cut-off points could be established, one for a grey zone, in which occasional spontaneous pregnancies could still be expected, and another below which a spontaneous pregnancy could only very occasionally be expected [29]. For normal sperm morphology, the normal cut-off value was $20 \%$ morphologically normal spermatozoa, compared with the $60 \%$ morphologically normal spermatozoa value proposed by MacLeod and Gold [16] and Eliasson [30]. The cut-off value for sperm morphology for the grey zone, as proposed by Van Zyl et al. [29], was 10\% morphologically normal spermatozoa and 3\% was the low cut-off value, below which spontaneous pregnancies were seldom seen $[29,31]$. These three categories are in agreement with the differentiation of semen analysis results into prognostic classes as published by Eliasson [30], although he used the terms normal, doubtful, pathological and severe pathological.

Similarly, in 1986 and 1988, Kruger et al. [32, 33] published data suggesting that three zones could be distinguished for sperm morphology with regard to the prediction of a males' fertility potential for in vitro fertilization (IVF) on the basis of percentage of the total number of oocytes fertilized per morphology interval group. The three cut-off values for these zones were $\geq 15 \%$ morphologically normal spermatozoa for a normal prognosis group, $5 \%-14 \%$ for the good prognosis or G-pattern group, and $\leq 4 \%$ for the poor prognosis or P-pattern group.

More recently, a number of papers have been published comparing sperm morphology cut-off values to in vivo pregnancy results in infertile populations or socalled fertile and subfertile populations (Table 2). EggertKruse et al. [34] published data from a prospective study including 89 randomly selected couples presenting for infertility investigations, for which the female partners were apparently normal. A spontaneous pregnancy rate of $20.2 \%(18 / 89)$ was observed after 1 year without treatment. An analysis of sperm morphology, as evaluated by

Table 2. Comparison of fifth World Health Organization (WHO) manual for normal morphology values with recently published literature values.

\begin{tabular}{|c|c|c|c|c|c|c|}
\hline \multirow{2}{*}{ Author } & \multirow{2}{*}{ Population $^{1}$} & \multicolumn{5}{|c|}{ Cut-off value } \\
\hline & & Fertile & Subfertile & Infertile & TZI & $\mathrm{AI}$ \\
\hline Van Zyl et al. $[23,29,31]$ & In vivo pregnancies & 20 & 10 & 3 & & \\
\hline Kruger et al. $[32,33]$ & IVF fertilization rates & & & & & \\
\hline Initial published intervals & & $\geq 15$ & $14-4$ & $\leq 3$ & & \\
\hline Intervals as used in practice & & $\geq 15$ & $14-5$ & $\leq 4$ & & \\
\hline Eggert-Kruse et al. [34] & In vivo pregnancies & 14 & 7 & 4 & & \\
\hline \multicolumn{7}{|l|}{ Ombelet et al. [35] } \\
\hline Tenth percentile & Fertile population & 10 & & & & \\
\hline ROC curve analysis & Fertile $v s$. subfertile & 5 & & & & \\
\hline Zinaman et al. [36] & Healthy couples & 8 & & & & \\
\hline Günalp et al. [38] & Fertile $v s$. subfertile & 12 & $5^{2}$ & & & \\
\hline \multicolumn{7}{|l|}{ Menkveld et al. [37] } \\
\hline ROC curve analysis & Fertile vs. subfertile & 4 & & & 1.64 & 8 \\
\hline Adjusted & Fertile vs. subfertile & 3 & & & 2.09 & 3 \\
\hline Tenth (upper) percentile & Fertile population & 2 & & & 1.82 & 5 \\
\hline Guzick et al. [39] & Fertile $v s$. subfertile & $>12$ & $9-12$ & $<9$ & & \\
\hline \multicolumn{7}{|l|}{ Haugen et al. [40] } \\
\hline Tenth percentile & Fertile population & 4 & & & & \\
\hline Fifth percentile & & 3 & & & 1.72 & \\
\hline \multicolumn{7}{|l|}{ Fifth WHO manual [1] } \\
\hline Fifth percentile & Recent fathers & 4 & & & & \\
\hline
\end{tabular}

Abbreviations: IVF, in vitro fertilization; ROC, receiver operating characteristic; TZI, teratozoospermia index; AI, acrosome index. ${ }^{1}$ See text for more detailed descriptions of populations investigated. ${ }^{2}$ Based on the positive and negative predictive values (see Gunalp et al. [38]). 
strict criteria, indicated three morphology cut-off points for the occurrence of a pregnancy, namely, $\geq 4 \%$ with a pregnancy rate of $21.5 \%(17 / 79), \geq 7 \%$ with a pregnancy rate of $27.4 \%(17 / 62)$ and $\geq 14 \%$ with a pregnancy rate of $34.3 \%(12 / 35)$.

Ombelet et al. [35] compared the semen parameters of 144 males from couples, in whom the wives had achieved a recent pregnancy within 12 months of unprotected intercourse. The subfertile group consisted of 143 consecutive men attending the infertility clinic during the same period, who did not achieve a pregnancy. Using the lower tenth percentile of the fertile population, the cut-off value for sperm morphology was $10 \%$ morphologically normal spermatozoa; using the receiver operating characteristic (ROC) curve analysis method, the cut-off value to distinguish between the fertile and subfertile group was 5\% morphologically normal spermatozoa.

Zinaman et al. [36] also conducted a prospective study with 210 healthy couples. The authors followed each couple for 12 menstrual cycles, during which the couple tried to conceive, and performed multiple semen analyses in each man over the period of exposure with known abstinence intervals. The couples were instructed to have intercourse as frequently as possible on and around the day of predicted ovulation. Semen analyses results were divided into pregnant and non-pregnant groups. The mean (SD) percentage of morphologically normal spermatozoa for the pregnant group was $6.2(3.7) \%$ and that for the nonpregnant group was $4.1(3.5) \%$. A decline in pregnancy rate was suggested below $8 \%$ morphologically normal spermatozoa.

Like Ombelet et al. [35], Menkveld et al. [37] compared the semen parameters of a fertile and subfertile population. The fertile population comprised a group of 107 healthy males without any history of fertility problems, the partners of whom had a spontaneous pregnancy within 1 year of unprotected intercourse and were pregnant at the time the husband was included into the study. A total of 103 males of couples attending the infertility clinic were included in the subfertile group. Using the ROC curve analysis, the cut-off point values for sperm morphology, teratozoospermia index (TZI) and the acrosome index (AI) were $4 \%$ morphologically normal spermatozoa, 1.64 and $8 \%$ normal acrosomes, respectively. Taking into consideration that the male contributes to the infertility problem in about $50 \%$ of cases, these values were recalculated in terms of false positive and false negative predictive values. The recalculation resulted in cut-off point values of $\leq 3 \%$ morphologically normal spermatozoa, a TZI value of $\geq 2.09$ and an AI value of $\leq 3 \%$ normal acrosomes. Taking the lower tenth percentile values of the fertile population, the cut-off points were $2 \%$ morphologically normal spermatozoa, $5 \%$ for the $\mathrm{AI}$ and a
TZI value of 1.82 .

Gunalp et al. [38] also published ROC curve analysis data of a prospective study that included 61 fertile males whose wives were at a gestational age of 8-23 weeks and 62 males attending the infertility clinic, resulting in a cutoff value of $12 \%$ morphologically normal spermatozoa. After an adjustment for the assumption of an infertility prevalence of $15 \%$ in the population, the cut-off point was calculated at 5\% morphologically normal spermatozoa.

Guzick et al. [39] also published a study compiling data from nine different centers representing 765 infertile and 696 fertile couples. By means of a classification and regression tree (CART) analysis, they found three predictive interval groups with regard to sperm morphology, namely, a fertile range when $>12 \%$ morphologically normal spermatozoa are present, an intermediate range of $9 \%-12 \%$ morphologically normal spermatozoa, indicating a reduced chance for conception, and a subfertile range when $<9 \%$ morphologically normal spermatozoa were present.

Haugen et al. [40] analyzed the semen samples of 99 men whose wives had recently achieved a pregnancy in 12 or fewer cycles of unprotected intercourse. Samples were only included if the period of abstinence was between 2 and 7 days. Both fifth and tenth percentiles were calculated for all semen parameters and resulted in cut-off values of 3\% and $4 \%$ morphologically normal spermatozoa, respectively.

The new proposed cut-off value in the fifth edition of the WHO manual is, therefore, in close agreement with recent published cut-off values, especially the value of $3 \%$ published by both Menkveld et al. [37] and Haugen et al. [40].

\section{Possible reasons for the decline in reference values}

The reason for the drastic decrease in normal sperm morphology cut-off values over the years, as illustrated in Figure 1, are mainly three-fold, namely, (i) the implementation of strict evaluation criteria with the unfortunate result that sperm morphology evaluation became overcritical with regard to normality, (ii) the fact that over the years more criteria for sperm morphological abnormalities were identified and introduced into the evaluation system, and (iii) a true decline because of negative environmental factors.

\subsection{Introduction of strict criteria}

The statement that, with strict evaluation, borderline spermatozoa should be regarded as abnormal, which is against the principle for the liberal approach that borderline spermatozoa should be regarded as normal, automatically placed a large number of spermatozoa, which would have been regarded as normal by the liberal approach, into the abnormal category when using the strict criteria approach, 
leading to an overall lower percentage of morphologically normal spermatozoa. This has been illustrated in several articles $[37,41]$.

Furthermore, the term 'strict' introduced a trend of over-criticality in the application of the definition of normality. An analysis of our own laboratory data indicated that the decline, as illustrated in Figure 1, was partly because of a real decline in normal sperm morphology and also because of stricter application of the criteria for normality of spermatozoa [42]. On the basis of the re-evaluation of 80 randomly selected semen smears from 1988 in 1995, the mean (SD) percentage of the 80 smears as evaluated in 1988 was $12.4(7.8) \%$ morphologically normal spermatozoa. The mean (SD) of the same 80 smears evaluated in 1995 was $9.6(5.5) \%$ morphologically normal spermatozoa, a decrease of 2.8 percentage points because of stricter application of the criteria over the period of 8 years. The overall mean percentage of morphologically normal spermatozoa in 1988 was also $12.4(7.6) \%(n=219)$ and decreased to a mean of $7.8(5.1) \%(n=227)$ in 1995 , showing overall decrease of 4.6 percentage points from 1988 to 1995 . Of the overall decrease of 4.6 percentage points from 1988 to 1995 , at least 2.8 percentage points could be attributed to a stricter approach in the evaluation of sperm morphology, and it can be postulated that the remaining 1.8 percentage points may be because of negative environmental factors.

This tendency toward a stricter approach over time was also illustrated in a publication by Horte et al. [43], in which a series of sperm morphology smears from 1980 to 1994 were re-evaluated. The results showed that the observed decline in the percentage of morphologically normal spermatozoa observed over the 15 -year period was mainly because of a stricter evaluation approach over the years. On the basis of the predictive values from a multiple regression analysis to calculate the end points of regression lines, the proportion of spermatozoa with normal morphology declined from $42.8 \%$ to $36.7 \%$ over the 15 -year period. The re-evaluation of the sampled smears only showed a minimal change, from $13.7 \%$ in 1980 to $13.3 \%$ in 1994 . The effect of the application of stricter criteria, as suggested by the consecutive WHO manuals, can be very clearly seen in the substantially lower means obtained with the re-evaluation of the sampled morphology smears.

\subsection{Introduction of additional criteria for sperm morpho- logy evaluation}

The original description of sperm abnormalities, as described, for example, by MacLeod and Gold [16], and Freund [44], concerned head abnormalities only. In 1971, Eliasson [30] introduced the concept that for a spermatozoon to be classified as normal, the whole sperm must be normal, including abnormalities of the neck/midpiece and tail, as well as the presence of excess cytoplasmic residue material. This automatically decreased the possible number of normal spermatozoa in a sample. As more sperm functions in the fertilization pathway [45] became known and these sperm functions could be associated with certain sperm morphology structures, the probability of sperm morphology abnormalities increased for a specific semen sample with the corresponding decrease in the mean percentage of morphologically normal spermatozoa. One of the more recently identified sperm morphology parameters based on sperm functionality includes that of the sperm acrosome. Acrosome size, such as too big or too small, and acrosome staining abnormalities are now important criteria for sperm morphology evaluation that had not been recognized until recently [46]. Thus, as more sperm functional abnormalities are recognized, a corresponding decrease in the percentage of morphologically normal spermatozoa will likely be observed.

\subsection{Negative environmental factors}

Carlson et al. [47] suggested a declining trend in semen quality worldwide. One of the main reasons for the suggested decline was the increasing effect of endocrine disruptors and other environmental polluters,

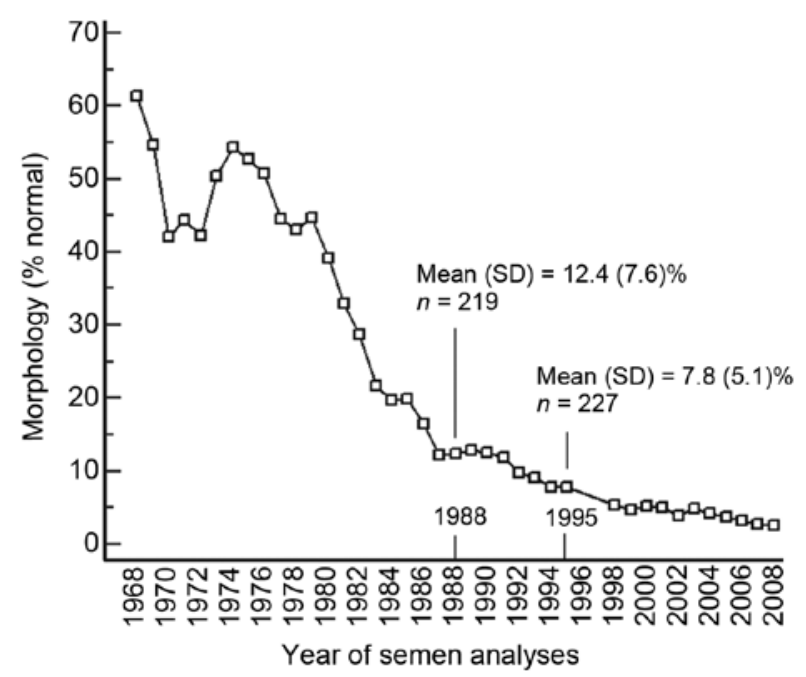

Figure 1. Decrease in mean morphology values over 39 years (1969-2008). The population consists of hospital patients with complaints of infertility who donated semen samples for evaluation at the Andrology Laboratory, Department of Obstetrics and Gynecology, Tygerberg Academic Hospital, South Africa. Since the beginning of 1971, sperm morphology evaluation and control have been performed by one person (RM). No conscious or deliberate changes in the evaluation criteria have been introduced since the mid-1970s, at which time strict evaluation criteria were introduced. Modified from Menkveld et al. [42]. 
such as insecticides and pesticides [48]. The entire issue of declining sperm parameters, including sperm morphology, has gained importance because of the recognition of several other trends that reflect a decline not only in semen production, but also in overall reproductive health, including the increase of testicular cancers and higher trends of cryptorchidism, together with a more general increase in testicular dysfunction [49], which also has a negative effect on normal sperm morphology. Another contributing factor may be a wider adoption of a more promiscuous lifestyle in some communities, leading to an increase in sexually transmitted diseases and male accessory gland infections (MAGI). The higher prevalence of MAGI, as identified by the presence of leukocytospermia, can also have a negative effect on spermatogenesis by reducing the percentage of morphologically normal spermatozoa and increasing the percentage of elongated spermatozoa [50].

\section{Additional tools to improve/refine sperm morphology evaluation}

By itself, the very low normal sperm morphology cut-off value proposed in the fifth edition of the WHO manual and also in the recently published articles by Menkveld et al. [37] and Haugen et al. [40] may be of limited prognostic value. However, over the years, the evaluation of sperm morphology has been refined and relies on more than just the percentage of morphologically normal spermatozoa. Additional sperm morphology parameters have been developed to improve the diagnostic abilities of sperm morphology parameters in the prediction of males' fertility potential. In males presenting with normal spermatozoa below the proposed WHO cut-off value of $\leq 4 \%$, two groups, or prognostic categories, can be identified from the morphology patterns and morphological abnormalities present.

The first group will have a poor prognosis because of mostly genetically determined sperm pattern defects, such as globozoospermia; short tail syndrome; spermatozoa with small heads, in most cases combined with very small acrosomes; cases of large-headed spermatozoa, mostly combined with the presence of large acrosomes; and the presence of large or small acrosomes alone. All of these types of abnormal spermatozoa have been associated with abnormal sperm functions and, thus, with poor in vivo and IVF rates.

The second group will include males with unspecified or non-genetically determined sperm defects or patterns caused by environmental factors, including medication.

\subsection{Genetically determined sperm defects \\ 5.1.1 Globozoospermia}

One of the best-known genetically determined sperm sterilizing defects or patterns is globozoospermia. These spermatozoa are easily recognizable by their small, roundhead form and the absence of acrosomes. Owing to the absence of acrosomes, the sperm head does not contain acrosin and is unable to bind and penetrate the zona pellucida. When couples are treated by intracytoplasmic sperm injection (ICSI), pregnancies can be achieved in some cases [51, 52].

\subsubsection{Short tail syndrome}

In some semen samples, the spermatozoa present with abnormalities of the spermatozoa tail and neck region. The most common defects are the stump or short tail syndrome, spermatozoa with dysplasia of the fibrous sheath, acephalic spermatozoa and spermatozoa with defects of the head-neck attachments [53]. In spermatozoa with neck attachment defects, the heads attach either to the tip or to the sides of the midpiece without an alignment with the sperm axis. This misalignment ranges from a complete lack of connection (difficult to observe) to a lateral positioning of the nucleus at a $90-180^{\circ}$ angle [53]. These patients have a poor prognosis, even with ICSI, and in cases in which fertilization has resulted, the pronuclei do not fuse, and zygotes fail to cleave and, thus, degenerate [53]. This sperm defect can be because of defective sperm aster formation, syngamy and cleavage. Even when sperm are carefully selected for the absence of these abnormalities using ICSI and positive human chorionic gonadotropin $(\beta \mathrm{HCG})$ levels are achieved, preclinical abortions are common [54].

\subsubsection{Small-headed spermatozoa}

Small-headed spermatozoa in men occur more frequently in couples complaining of infertility than currently diagnosed because of the unfortunate fact that this condition is not commonly recognized and reported as a severe sperm abnormality. This abnormality is stable over time. When recognized, small-headed spermatozoa are easy to observe, because most spermatozoa are small (head length $<3.5 \mu \mathrm{m}$ and head width $<2.5 \mu \mathrm{m}$ with Diff-Quik staining) [55]. These spermatozoa may also present with very small, abnormally formed acrosomes. Individuals who are not diagnosed with this condition will achieve poor results with IVF and even with ICSI, but by selecting the more normally appearing spermatozoa, ICSI results can be improved. Even when larger spermatozoa with small acrosomes are present, with $<30 \%$ of normal sperm head size, the prognosis for the patient with IVF is poor, because these spermatozoa have a low vitality and are not able to undergo the acrosome reaction [56].

\subsubsection{Large-headed spermatozoa}

These spermatozoa present with severely abnormal 
megalo heads and multiple tails, and are observed in the ejaculate and testicular biopsies [57]. Low fertilization and pregnancy rates are achieved with ICSI, which may be because of the high incidence of chromosomal aberrations in the ejaculated megalo-headed spermatozoa [58].

\subsection{Non-genetic sperm morphology aberrations}

The second group, with unspecified or non-genetically determined sperm defects or patterns caused by environmental or other stress factors, including medication, differs from the group with genetically determined permanent sperm defects, which will not respond to treatment. Sperm defects in the second group can return to normal or disappear when the source of the alternations or stress is removed or treated. Males with these types of sperm abnormalities still have a good chance for normal in vivo conception or, alternatively, a good prognosis for IVF without ICSI. To further enhance the diagnosis of men with a potentially good IVF chance, several additional morphology tools are available, such as the TZI, AI, the presence of cytoplasmic residues and sperm head measurements.

\subsubsection{Megalo heads}

Megalo heads can also be caused by the use of medicine, such as sulfasalazine, for the treatment of ulcerative colitis [59] and Crohn's disease [60]. When sulfasalazine treatment is stopped, the semen parameters can return to normal values, and the megalo heads can disappear. It is not clear whether this will result in fertilization and viable pregnancies. However, the substitution of sulfasalazine with mesalazine has resulted in improved semen quality and the reduction of the megalo heads, subsequently resulting in viable pregnancies [61].

\subsubsection{Elongated spermatozoa}

Elongation is generally recognized as a stress-induced sperm morphology aberration, and is prevalent especially in MAGI and in the presence of a varicocele [62]. The classic tapering or narrow form has been described in detail by Eliasson [30] as being a sperm head longer than $5 \mu \mathrm{m}$ with a width $<3 \mu \mathrm{m}$ or a length of $<5 \mu \mathrm{m}$ and a width of $<2 \mu \mathrm{m}$. Pyriform heads are also included under elongation of spermatozoa. Sperm elongation is accompanied by severe structural as well as DNA damage. The increased sperm head length results from an abnormally elongated nucleus, which also presents particular membranous layers between the outer and inner leaves of the nuclear envelope. The sperm nuclear anomalies are also associated with anomalies of the neck region, persistence of cytoplasmic residual material and an increased frequency of chromosomal aneuploidies, together with impaired chromatin compaction, possibly because of meiotic non-disjunction during spermatogenesis [63]. Low ICSI fertilization rates have also been found in men with severely elongated spermatozoa compared with other sperm morphology abnormalities [64]. However, when the MAGI is treated with long-term antibiotics or the varicocele is treated with a varicocelectomy, semen characteristics, including sperm morphology, can improve and pregnancies can result [65]. In case of varicocelectomies, in which no substantial improvement in semen quality is observed, pregnancies do still occur because sperm DNA quality improves because of the reduction of ROS production following the varicocelectomy [66].

\subsubsection{Cytoplasmic residues}

In addition to abnormalities of the head, neck/midpiece and tail, the fourth major sperm abnormality is the presence of cytoplasmic material at the neck/midpiece region and sometimes on the tails of spermatozoa. The presence, size and terminology of the cytoplasmic droplets or cytoplasmic residues are controversial. Originally, Eliasson [30], in the 1980 and 1987 WHO manuals [6, 7], and Menkveld et al. [12] indicated that a normal cytoplasmic droplet present on spermatozoa should be $<50 \%$ of a normal sperm head. This was changed to $<30 \%$ in the 1999 WHO manual [9]. Recently, Cooper et al. [67] and Cooper [68] addressed the issue of the presence and size of cytoplasmic bodies, as well as the correct terminology to be used. According to Cooper [68], it is clear that retention of cytoplasmic material on spermatozoa, as seen in air dried and stained semen smears, can be associated with impaired sperm function. It is clear from this article and from our own experience [69] that no amount of cytoplasmic material should be present on a normal spermatozoon and, if observed, it should be regarded as an abnormality, regardless of the size or amount of cytoplasmic material present. Cooper [68] also suggested that the correct term to be used for the presence of cytoplasmic material should be 'excess cytoplasmic residues' or cytoplasmic residues. The presence of cytoplasmic residues is associated with sperm immaturity and especially the production of ROS, with subsequent sperm DNA damage; although IVF pregnacies may occur, decreased pregnancy rates are reported [70]. Therefore, much more attention should be given to the presence of cytoplasmic residues with the routine evaluation of sperm morphology.

\subsubsection{Sperm measurements}

The earlier sections showed that sperm size, and therefore sperm measurement, is a very important aspect of the sperm morphology evaluation process. However, the measurements proposed by Eliasson [30], and adopted in the WHO manuals [6-9] and other publications [12] are in need of re-evaluation, because the range allowed, 
especially the normal head length of $3.0-5.0 \mu \mathrm{m}$, is too wide. Our own experience indicates that the head length for normal spermatozoa may vary between 4.0 and $4.5 \mu \mathrm{m}$, with a mean of $4.07 \pm 0.19 \mu \mathrm{m}$ and a mean width of $2.98 \pm$ $0.14 \mu \mathrm{m}$, as measured with a built-in microscope eye-piece micrometer [27]. We have shown in several publications that males presenting with large-headed spermatozoa of $>5.0 \mu \mathrm{m}$ in length, and proportional increases in width and/or large acrosomes, as seen in Papanicolaou-stained smears, are associated with poor IVF results [32] and decreased sperm functional abilities [46].

Alternative measurements have been proposed by several authors, such as Katz et al. in 1986 [71] and Garrett and Baker in 1995 [72], who reported length/ width measurements of $4.37 \mu \mathrm{m}$ by $2.83 \mu \mathrm{m}$ and $4.35 \mu \mathrm{m}$ by $2.89 \mu \mathrm{m}$ for Papanicolaou- and Shorr-stained smears of donors, respectively. The new WHO manual no longer refers to the 'old' sperm measurements, but states that head dimensions of 77 Papanicolaou-stained semen smears measured with a computerized system, have a median length of $4.1 \mu \mathrm{m}(3.7-4.7 \mu \mathrm{m}, 95 \% \mathrm{CI})$ and width of $2.8 \mu \mathrm{m}(2.5-3.2 \mu \mathrm{m})$ and a length-to-width ratio of 1.5 (1.3-1.8). Unfortunately, the manual also states that 'once a normally shaped spermatozoa is identified, an eye-piece micrometer may be useful for distinguishing between the normal and abnormal (size - own word) spermatozoa, but with this technique the form of the sperm head is much more important than its dimension unless grossly abnormal.' However, sperm measurements are of utmost importance for the normal oval form. Sperm measurement is an area deserving much more attention in today's sperm morphology assessment methodology, and more research is needed to establish more accurate sperm measurements.

\subsubsection{Detection of leukocytes}

Although not actually a sperm morphology parameter, determination of the presence of leukocytes, especially polymorphonuclear leukocytes, is an important aspect of the sperm morphology evaluation procedure [65]. Polymorphonuclear leukocytes are the main source of ROS production in semen, leading to increased sperm DNA damage. Although sometimes reported as part of the morphology evaluation procedure, much more emphasis should be placed on the presence of polymorphonuclear leukocytes in semen and the importance of antibiotic treatment for leukocytospermia and the subsequent possible improvement in pregnancy outcome $[65,70]$.

\section{$5.3 \mathrm{TZI}$}

The two most recent WHO manuals $[8,9]$ recommended that spermatozoa should only be classified as normal or abnormal and that a note should be made if a specific abnormality occurs with a frequency of $>20 \%$.
However, an abnormal spermatozoon may have only one specific abnormality or any combination of two to four abnormalities, as described above. The TZI was introduced to reflect the mean number of abnormalities per abnormal spermatozoon.

The usefulness of the TZI seems to be limited, and it is seldom used in the literature as a tool in decision-making with regard to possible treatment of patients because it does not indicate a specific dominant abnormality present in a specific semen sample. However, a high TZI value of $\geq 1.90$ can be regarded as a poor prognosis for normal IVF, and these patients should be taken directly to ICSI [73]. Following a literature search and on the basis of an analysis of our own data, we concluded that the TZI has little predictive value for in vivo and IVF outcomes [74].

\subsection{Acrosome index (AI)}

The AI is an indication of the percentage of spermatozoa with morphologically normal acrosomes present in a semen sample and can be regarded as a reflection of the functional ability of the spermatozoa, because the AI is based on the morphological appearance of the acrosomes with regard to size (too small or too large), staining properties and form (oval or amorphous shaped).

Incorporation of the AI in the sperm morphology evaluation process is, therefore, an important tool in the prediction of fertilization outcome, especially in cases of severe teratozoospermia (now $\leq 4 \%$ normal forms). The AI was found to be a more sensitive predictor of expected IVF rates compared with normal sperm morphology, whereas the TZI was a less sensitive predictor compared with normal sperm morphology, on the basis of an ROC curve analysis for the prediction of a $>37 \%$ IVF rate with areas under the curve of $0.927,0.712$ and 0.653 for the AI, percentage of normal morphology and TZI values, respectively [75].

\section{Future aspects of sperm morphology evaluation}

The results of sperm morphology evaluations can be further improved by better international standardization for the whole sperm morphology evaluation procedure, better international training and improvement, and standardization of international external quality control (EQC) schemes.

\subsection{International standardization of morphology evalua- tion procedures}

According to the literature, although many authors mention in their articles that strict criteria/1999 WHO criteria have been used for sperm morphology evaluation in their laboratories, many may still use wet preparations, Diff-Quik-stained smears or other staining methods, 
contributing to confusion in the literature and diverse results obtained for sperm morphology evaluation in different laboratories throughout the world [76]. Different staining procedures can lead to different sperm morphology evaluation results [77].

Therefore, before comparisons of the results of different laboratories can be made, large-scale standardization should be implemented in laboratories worldwide as far as preparation and staining techniques are concerned, focusing on well-prepared smears and staining according to the Papanicolaou method only.

\subsection{Standardization of international external quality control schemes}

The lack of standardization in the EQC schemes is illustrated in the article by Cooper et al. [78], in which the results of three EQC programs for the same semen smears were compared, namely, those of the ESHRE SIG-A, the EAA, and the UKNEQAS schemes. Assessment by the SIG-A ECQ was stricter than that of the other two programs, and the results of the UKNEQAS were much more widely distributed compared with the EAA scheme results.

\subsection{International cooperation in standardization}

The users of different international EQC programs should start to work together by interchanging standardized QC material and then, after a trial period, come together to discuss the results and to try to standardize their interpretations for sperm morphology evaluations on the basis of strict evaluation principals.

\section{Conclusions}

The very low cut-off value for sperm morphology of $4 \%$ morphologically normal spermatozoa, as proposed in the new WHO manual [1], is in agreement with recently published values $[37,40]$ and with the trend of declining mean normal sperm morphology values reported in the literature [34-40].

On its own, the new proposed very low normal value may not provide a strong predictive value for a male's fertility potential, as originally reported for sperm morphology evaluated according to strict criteria [79]. However, a good predictive value can still be obtained if the holistic strict approach for sperm morphology evaluation is followed and used with the additional sperm morphology parameters available in cases in which severe teratozoospermia ( $\leq 4 \%$ normal) is present. In these cases, it becomes of utmost importance that clinicians receive more detailed data on abnormal sperm patterns and specific abnormalities so they may use the additional information to make a final decision regarding the treatment of the patient, because certain morphology patterns and sperm abnormalities are known to be of strong prognostic value.

The normal WHO value for sperm morphology is based on Papanicolaou-stained smears. Another set of normal cut-off values needs to be established for rapid staining methods because the literature indicates that higher normal values are obtained in Diff-Quik-stained semen samples compared with the same samples stained with the Papanicolaou method [77].

A high percentage of morphologically abnormal spermatozoa can be associated with the presence of poor sperm functionality. On the other hand, morphologically normal-appearing spermatozoa should also be further investigated for normal sperm function and DNA content because morphological normality does not necessarily imply normal sperm function $[80,81]$.

In addition, better international standardization of the technical methodology, consensus on the interpretation of sperm morphology evaluation criteria, and standardized international EQC schemes are of the utmost importance.

\section{Acknowledgment}

Statistical analyses, calculations and the creation of graphs included in this article were performed with the MedCalc ${ }^{\circledR}$ statistical software package, Version 10.0.1.0 (MedCalc Software, Broekstraat 52, 9030 Mariakerke, Belgium).

\section{References}

1 World Health Organisation. WHO Laboratory Manual for the Examination and Processing of Human Semen, 5th ed. Geneva: World Health Organization; 2010.

2 Menkveld R, Kruger TF. Evaluation of sperm morphology by light microscopy. In: Acosta AA, Kruger TF, editors. Human Spermatozoa in Assisted Reproduction. Carnforth: Parthenon Publishing; 1996, p89-107.

3 Cary HW. Examination of semen with reference to gynaecological aspects. Am J Obstet Dis Women Child 1916; 74: 615-38.

4 Williams WW, Salvage A. Observations on the seminal micropathology of bulls. Cornell Vet 1925; 15: 353-75.

5 Moench GL, Holt H. Sperm morphology in relation to fertility. Am J Obstet Gynecol 1931; 22: 199-210.

6 World Health Organization. WHO laboratory manual for the examination of human semen and semen-cervical mucus interaction, 1st edn. Singapore: Press Concern; 1980.

7 World Health Organization. WHO laboratory manual for the examination of human semen and semen-cervical mucus interaction, 2nd edn. Cambridge: Cambridge University Press; 1987.

8 World Health Organization. WHO laboratory manual for the examination of human semen and sperm-cervical mucus interaction, 3rd edn. Cambridge: Cambridge University Press; 1992.

9 World Health Organization. WHO laboratory manual for the examination of human semen and sperm-cervical mucus interaction, 
4th edn. Cambridge: Cambridge University Press; 1999.

10 Comhaire F, Schoonjans F, Vermeulen L, De Clercq N. Methodological aspects of sperm morphology evaluation: comparison between strict and liberal criteria. Fertil Steril 1994; 62: 857-61.

11 Menkveld R. An investigation of environmental influences on spermatogenesis and semen parameters. [PhD dissertation (in Afrikaans)]. South Africa: Faculty of Medicine, University of Stellenbosch; 1987.

12 Menkveld R, Stander FS, Kotze TJ, Kruger TF, van Zyl JA. The evaluation of morphological characteristics of human spermatozoa according to stricter criteria. Human Reprod 1990; 5: 586-92.

13 Mortimer D, Menkveld R. Sperm morphology assessmenthistorical perspectives and current opinions. J Androl 2001; 22: 192-205.

14 Hartmann GC, Schoenfeld C, Copeland E. Individualism in the seminal picture of infertile men. Fertil Steril 1964; 15: 231-53.

15 Hellinga G. Clinical Andrology. London: William Heineman Medical Books Ltd; 1976.

16 MacLeod J, Gold RZ. The male factor in fertility and infertility. IV. Sperm morphology in fertile and infertile marriage. Fertil Steril 1952; 2: 394-414.

17 Page EW, Holding F. The clinical interpretation of 1000 semen analyses among applicants for sterility studies. Fertil Steril 1951; 2: 140-51.

18 Menkveld R, Franken DR, Kruger TF, Oehninger S. Sperm selection capacity of the human zona pellucida. Mol Reprod Dev 1991; 30: 346-52.

19 Liu DY, Baker HW. Morphology of spermatozoa bound to the zona pellucida of human oocytes that failed to fertilize in vitro. J Fertil Reprod 1992; 94: 71-84.

20 MacLeod J. Human seminal cytology following the administration of certain antispermatogenic compounds. In: Austin CR, Perry JS, editors. Agents Affecting Fertility. London: Churchill; 1965, p93-123.

21 Eliasson R. Analysis of semen. In: Behrman SJ, Kistner SW, editors. Progress in Infertility, 2nd edn. Boston: Little Brown Co.; 1975. p691.

22 Van Zyl JA, Menkveld R, Kotze TJ, Retief AE, Van Niekerk WA. Oligozoospermia: a seven-year survey of the incidence, chromosomal aberrations, treatment and pregnancy rate. Int $\mathrm{J}$ Fertil 1975; 20: 129-32.

23 Van Zyl JA, Menkveld R, Kotze TJ, van Niekerk WA. The importance of spermiograms that meet the requirements of international standards and the most important factors that influence semen parameters. In: Proceedings of the 17th Congress of the International Urological Society. Paris: Diffusion Dion Editeurs; 1976; 2: 263.

24 Menkveld R, Kruger TF. Basic semen analysis. In: Acosta AA, Swanson RJ, Ackerman SB, Kruger TF, Van Zyl JA, et al, editors. Human Spermatozoa in Assisted Reproduction. Balitimore: Williams and Wilkins; 1990. p68.

25 Menkveld R, Kruger TF. Basic semen analysis. The Tygerberg experience. In: Acosta AA, Swanson RJ, Ackerman SB, Kruger TF, Van Zyl JA, et al, editors. Human Spermatozoa in Assisted Reproduction. Baltimore: Williams and Wilkins; 1990. p164.

26 Menkveld R, Kruger TF. Basic semen analysis. In: Acosta AA, Kruger TF, editors. Human Spermatozoa in Assisted Reproduction, 2nd edn. Carnforth: Parthenon Publishing; 1996. p53.

27 Menkveld R. The basic semen analysis. Chapter 9. In:
Oehninger S, Kruger TF, editors. Male Infertility. Diagnosis and Treatment. Oxon: Informa Healthcare. 2007. p141.

28 Cooper TG, Noonan E, von Eckardstein S, Auger J, Baker HW, et al. World Health Organization reference values for human semen characteristics. Hum Reprod Update 2009 Nov 24. [Epub ahead of print].

29 Van Zyl JA, Kotze TJ, Menkveld R. Predictive value of spermatozoa morphology in natural fertilization. In: Acosta AA, Swanson RJ, Ackerman SB, Kruger TF, van Zyl JA, et al, editors. Human Spermatozoa in Assisted Reproduction, Baltimore: Williams and Wilkins; 1990, p319-24.

30 Eliasson R. Standards for investigation of human semen. Andrologie 1971; 3: 49-64.

31 Van Zyl JA, Menkveld R. Oligozoospermia: recent prognosis and outcome of 73 pregnancies in oligozoospermia couples. Andrologia 2006; 38: 87-91.

32 Kruger TF, Menkveld R, Stander FS, Lombard CJ, Van der Merwe JP, et al. Sperm morphological features as a prognostic factor in in vitro fertilization. Fertil Steril 1986; 46: 1118-23.

33 Kruger TF, Acosta AA, Simmons KF, Swanson RJ, Matta JF, et al. Predictive value of abnormal sperm morphology in in vitro fertilization. Fertil Steril 1988; 49: 112-7.

34 Eggert-Kruse W, Schwarz H, Rohr G, Demirakca T, Tilgen W, et al. Sperm morphology assessment using strict criteria and male fertility under in-vivo conditions of conception. Hum Reprod 1996; 11: 139-46.

35 Ombelet W, Bosmans E, Janssen M, Cox A, Vlasselaer J, et al. Semen parameters in a fertile versus subfertile population: a need for a change in the interpretation of semen testing. Hum Reprod 1997; 12: 987-93.

36 Zinaman MJ, Brown CC, Selevan SG, Clegg ED. Semen Quality and human fertility: a prospective study with health couples. J Androl 2000; 21: 145-53.

37 Menkveld R, Wong WY, Lombard CJ, Wetzels AM, Thomas CM, et al. Semen parameters including WHO and strict criteria morphology, in a fertile and subfertile population: an effort towards standardisation of in vivo thresholds. Hum Reprod 2001; 16: 1165-71.

38 Gunalp S, Onculoglu C, Gurgan T, Kruger TF, Lombard CJ. A study of semen parameters with emphasis on sperm morphology in a fertile population: an attempt to develop clinical thresholds. Hum Reprod 2001; 16: 110-4.

39 Guzick DS, Overstreet JW, Factor-Litvak P, Brazil CK, Nakajima ST, et al. Sperm morphology, motility and concentration in fertile and infertile men. N Engl J Med 2001; 345: 1388-93.

40 Haugen TB, Egeland T, Magnus O. Semen parameters in Norwegian fertile men. Int J Androl 2006; 27: 66-71.

41 Harrison KL, Harrison M. Predictive ability of strict and WHO sperm morphology criteria for in vitro fertilization. Aust J Med Sci 1996; 17: 144-5.

42 Menkveld R, van Zyl JA, Kotze TJ, Joubert G. Possible changes in male fertility over a 15 -year period. Arch Androl $1986 ; 17143-4$.

43 Horte A, Vierula M, Toppari J, Suominen J. Reassessment of sperm morphology of archival semen smears from the period 1980-1994. Int J Androl 2001; 24: 120-4.

44 Freund $M$. Standards for the rating of human sperm morphology. Int J Fertil 1966; 11: 97-118.

45 Wassarman PM. Fertilization in mammals. Sci Am 1988; 259: 52-8.

46 Menkveld R, Rhemrev JP, Franken DR, Vermeiden JP, Kruger TF. Acrosomal morphology as a novel criterion for male fertility diagnosis: relation with acrosin activity, morphology (strict 
criteria) and fertilization in vitro. Fertil Steril 1996; 65: 637-44.

47 Carlson E, Giwercman A, Keiding N, Skakkebaek N. Evidence for declining quality of semen during past 50 years. Br Med J 1992; 305: 609-13.

48 Safe S. Clinical correlates of environmental endocrine disruptors. Trends Endocrinol Metab 2005; 16: 139-44.

49 Swan SH, Elkin EP, Fenster L. The question of declining sperm density revisited: an analysis of studies published 1934-1996. Environ Health Perspect 2000; 108: 961-6.

50 Menkveld R. Male accessory gland infection as a possible additional cause for the declining trend in semen parameter values. Int J Androl 2008; 31 (Suppl 1): 29 (Abstract).

51 Coetzee K, Windt ML, Menkveld R, Kruger TF, Kitshoff M. Short communication: an intracytoplasmic sperm injection with a globozoospermic male. J Assist Reprod Genet 2001; 18: 311-3.

52 Baccetti B, Capitani S, Collodel G, Di Cairano G, Gambera L, et al. Genetic sperm defects and consanguinity. Hum Reprod 2001; 16: 1365-71.

53 Chemes HE. Phenotypes of sperm pathology: genetic and acquired forms in infertile men. J Androl 2000; 21: 799-808.

54 Rawe VY, Terada Y, Nakamura S, Chillik CF, Brugo Olmedo S, et al. A pathology of sperm centriole responsible for defective sperm aster formation, syngamy and cleavage. Hum Reprod 2002; 17: 2344-9.

55 Kihaile P, Hirotsuru K, Kumasako Y, Misumi J, Utsunomiya T. Fertilization rates of small-headed sperm in conventional IVF and ICSI. Arch Androl 2003; 49: 327-9.

56 Menkveld R, El-Garem Y, Schill WB, Henkel R. Relationship between human sperm acrosomal morphology and acrosomal function. J Assist Reprod Genet 2003; 20: 432-8.

57 Kahraman S, Akarsu C, Cengiz G, Dirican K, Sözen E, et al. Fertility of ejaculated and testicular megalohead spermatozoa with intracytoplasmic sperm injection. Hum Reprod 1999; 14: 726-30.

58 Chelli MH, Albert M, Ray PF, Guthauser B, Izard V, et al. Can intracytoplasmic morphologically selected sperm injection be used to select normal-sized sperm heads in infertile patients with macrocephlic sperm head syndrome? Fertil Steril 2008 Dec 3. [Epub ahead of print].

59 Toth A. Reversible effect of salicylazosulfapyridine on semen quality. Fertil Steril 1979; 31: 538-40.

60 Cosentino MJ, Chey WY, Takihara H, Cockett AT. The effects of sulfasalazine on human male fertility potential and seminal prosdaglandins. J Urol 1984; 132: 682-6.

61 Riley SA, Lecarpentier J, Mani V, Goodman MJ, Mandal BK, et al. Sulphasalazine induced seminal abnormalities in ulcerative colitis: results of mesalazine substitution. Gut 1987; 28: 1008-12.

62 Menkveld R, Kruger TF. Sperm morphology and male urogenital infections. Andrologia 1998; 30 (Suppl 1): 49-53.

63 Prisant N, Escalier D, Soufir JC, Morillion M, Schoevaert D, et al. Ultrastructural nuclear defects and increased chromosome aneuploidies in spermatozoa with elongated heads. Hum Reprod 2007; 22: 1052-9.

64 Osawa Y, Sueoka K, Iwata S, Shinohara M, Kobayashi N, et al. Assessment of the dominant abnormal form is useful for predicting the outcome if intracytoplasmic sperm injection in the case of sever teratozoospermia. J Assist Reprod Genet 1999; 16: 436-42.
65 Menkveld R. Leukocytospermia. In: Daya S, Harrison RF, Kempers RD, editors. Advances in Fertility and Reproductive Medicine. International Congress Series 1266. Amsterdam: Elsevier B.V; 2004, p218-24.

66 Nasr-Esfahani MH, Abasi A, Razavi S, Ashrafi S, Tavalaee M. Varicocelectomy: semen parameters and protamine deficiency. Int J Androl 2009; 32: 115-22.

67 Cooper TG, Yeung LH, Fetic S, Sobhani A, Nieschlag E. Cytoplasmic droplets are normal structures of human sperm but are not well preserved by routine procedures for assessing sperm morphology. Hum Reprod 2004; 19: 2283-8.

68 Cooper TG. New debate. Cytoplasmic droplets: the good the bad or just confusing? Hum Reprod 2005; 20: 9-11.

69 Rhemrev JP, Menkveld R, Rooseboom TJ, Van Overveld FW, Teerlink T, et al. The acrosome index and fast TRAP as projection of IVF outcome. Hum Reprod 2001; 16: 1885-92.

70 Henkel R, Hajimohammad M, Stalf T, Hoogendijk C, Mehnert C, et al. Influence of deoxyribonucleic acid damage on fertilization and pregnancy. Fertil Steril 2004; 81: 965-72.

71 Katz DE, Overstreet JW, Samuals SJ, Niswander PW, Bloom $\mathrm{TD}$, et al. Morphometric analysis of spermatozoa in the assessment of human male fertility. J Androl 1986; 7: 203-10.

72 Garrett C, Baker WH. A new fully automated system for the morphometric analysis of human sperm heads. Fertil Steril 1995; 63: 1306-17.

73 Rowe PJ, Comhaire FH, Hargreave TB, Mahmoud AM. WHO manual for the standardized investigation, diagnosis and management of the infertile male. Cambridge: Cambridge University Press; 2000, p52-4.

74 Menkveld R. The teratozoospermia index: a useful sperm morphology parameter for inclusion in the new WHO semen evaluation manual? Andrologie 2006; 16: 357 (Abstract).

75 Menkveld R, Stander FS, Kruger TF. Comparison between acrosome index and teratozoospermia index as additional criteria to sperm morphology in the prediction of expected invitro fertilisation outcome. Hum Reprod 1998; 13 (Abstract Book 1): 52.

76 Ombelet W, Pollet H, Bosmans E, Vereecken A. Results of a questionnaire on sperm morphology assessment. Human Reprod 1997; 12: 1015-20.

77 Henkel R, Schreiber G, Sturmhoefel A, Hipler UC, Zermann $\mathrm{H}$, et al. Comparison of three staining methods for the morphological evaluation of human spermatozoa. Fertil Steril 2008; 89: 449-55.

78 Cooper TG, Björndahl L, Vreeburg J, Nieschlag E. Semen analysis and external quality control schemes for semen analysis need global standardization. Int J Androl 2002; 25: 306-11.

79 Coetzee K, Kruger TF, Lombard CJ. Predictive value of normal sperm morphology: a structural literature review. Hum Reprod Update 1998; 4: 73-82.

80 Liu DY, Clarke GN, Martic M, Garrett C, Baker HW. Frequency of disordered zona pellucida (ZP)-induced acrosome reaction in infertile men with normal semen analysis and normal spermatozoa-ZP binding. Hum Reprod 2001; 16: 1185-90.

81 Liu DY, Garrett G, Baker HW. Low proportion of sperm can bind to the zona pellucida of human oocytes. Hum Reprod 2003; 18: 2382-9. 